\title{
Impact of de-escalation therapy on clinical outcomes for intensive care unit-acquired pneumonia
}

Mi Kyong Joung ${ }^{1}$, Jeong-a Lee ${ }^{2}$, Soo-youn Moon ${ }^{3}$, Hae Suk Cheong ${ }^{4}$, Eun-Jeong Joo ${ }^{1}$, Young-Eun Ha', Kyung Mok Sohn ${ }^{5}$, Seung Min Chung ${ }^{1}$, Gee Young Suh ${ }^{6}$, Doo Ryeon Chung ${ }^{1}$, Jae-Hoon Song ${ }^{1,7}$ and Kyong Ran Peck ${ }^{1 *}$

\begin{abstract}
Introduction: De-escalation therapy is a strategy currently used for the management of nosocomial pneumonia. In this study, we evaluated clinical outcomes and risk factors related to de-escalation therapy in patients with intensive care unit (ICU)-acquired pneumonia.

Methods: This was a retrospective observational cohort study of ICU patients who developed pneumonia more than 48 hours after admission to the ICU at Samsung Medical Center from September 2004 to December 2007.

Results: The 137 patients comprised 44 (32.1\%) who received de-escalation therapy and 93 in the non-deescalation group. The de-escalation group showed a lower pneumonia-related mortality rate than the non-deescalation group by day $14(2.3 \%$ vs. $10.8 \%$, respectively; $P=0.08)$ and by day $30(2.3 \%$ vs. $14 \%$, respectively; $P=$ 0.03 ) after the diagnosis of pneumonia. The variables independently associated with ICU-acquired pneumoniarelated mortality included the Acute Physiology and Chronic Health Evaluation II (APACHE II) score and the modified Clinical Pulmonary Infection Score (CPIS) after 5 days with pneumonia. The non-de-escalation group had significantly higher APACHE II score and modified CPIS after 5 days with ICU-acquired pneumonia compared to the de-escalation group. Among all patients, 20.4\% (28 of 137) had negative cultures for pathogens, and $42.9 \%$ (12 of 28) received de-escalation therapy. The latter 12 patients received de-escalation therapy and survived 30 days after the diagnosis of pneumonia.

Conclusions: Patients in the de-escalation group showed a significantly lower mortality rate compared to patients in the non-de-escalation group. De-escalation therapy can be safely provided to patients with ICU-acquired pneumonia if they are clinically stable by day 5, even in those whose respiratory specimen cultures yield no specific pathogens.
\end{abstract}

\section{Introduction}

Nosocomial pneumonia accounts for almost one-half of all intensive care unit (ICU) mortality and approximately $60 \%$ of mortality due to all nosocomial infections. The initial choice of antimicrobial therapy is critical to the clinical outcome of patients with nosocomial pneumonia. Early and aggressive empirical therapy with broadspectrum agents targeted at the likely pathogens has been associated with a reduction in the ventilator-

\footnotetext{
* Correspondence: krpeck@skku.edu

'Division of Infectious Diseases, Samsung Medical Center, Sungkyunkwan University School of Medicine, 50 Ilwon-dong, Gangnam-gu, Seoul 135-710, Republic of Korea

Full list of author information is available at the end of the article
}

associated pneumonia (VAP) mortality rate [1-9]. Awareness of the need for early and appropriate therapy, however, may tempt the clinician to use aggressive empirical therapy at the first sign of infection. Such empirical practices could create a vicious cycle of early and aggressive broad-spectrum antibiotic therapy that may in turn lead to overuse of antibiotics and an increase in antimicrobial resistance.

De-escalation therapy is a method currently used for the management of serious infections, especially in nosocomial pneumonias [2,10-17]. Early administration of broad-spectrum antibiotics has been used for treatment to improve appropriate use of empirical therapy.

\section{Biomed Central}


Sequential de-escalation provides maximum benefit for the individual patient and reduces the selection pressure fueling the development of resistance. De-escalation strategies provide clinical balance between using broadspectrum empirical antimicrobial agents and delaying the initiation of targeted therapy pending the bacteriological culture results. Several studies have shown that deescalation therapy leads to reduced antibiotic use, shorter duration of therapy and reduced mortality $[12,13,17]$.

The most recent VAP treatment guidelines of the American Thoracic Society (ATS) and the Infectious Diseases Society of America (IDSA) include recommendations for early, appropriate, broad-spectrum coverage and subsequent de-escalation of antibiotic regimens when possible, based on microbiological culture findings [18].

ATS and IDSA VAP treatment guidelines have suggested that negative lower respiratory tract cultures can be used to discontinue antibiotic therapy in a patient who shows clinical improvement at 48 to 72 hours after the diagnosis of pneumonia and has cultures obtained, in the absence of antibiotic initiation or change, over the previous 72 hours $[12,15,18]$. However, the outcome of de-escalation therapy in patients with negative cultures has not previously been reported.

Studies of de-escalated antimicrobial therapy based on antimicrobial sensitivity testing of microbiological cultures have reported that de-escalation therapy is not possible in patients with negative cultures [14]; that is, the outcome of patients with ICU-acquired pneumonia with negative cultures is unfavorable.

In this study, we evaluated pneumonia-related mortality at day 14 after diagnosis of pneumonia and the risk factors associated with pneumonia-related mortality among patients with ICU-acquired pneumonia that were managed with de-escalation therapy. Furthermore, we focused on the outcome of de-escalation therapy in patients with ICU-acquired pneumonia who had negative cultures.

\section{Materials and methods}

\section{Study design and patients}

This retrospective observational cohort study was conducted in 30-bed medical and surgical ICUs at Samsung Medical Center, a tertiary care university hospital, from September 2004 to December 2007. Patients were enrolled in the study if they were at least 18 years of age and the physicians established a diagnosis of ICUacquired pneumonia that occurred more than 48 hours after admission to the ICU that required antibiotic treatment. Data collected included patient demographics, underlying disease, hospital and ICU admission dates, diagnosis at the time of ICU admission, chest radiographic findings, microbiological cultures, antimicrobial therapy prior to and during the ICU stay, duration of mechanical ventilation prior to and after the diagnosis of pneumonia and severity-of-illness indices, including the Acute Physiology and Chronic Health Evaluation II (APACHE II) score [19], the modified clinical pulmonary infection score (CPIS) [20] and the Charlson comorbidity index score (CCS) [21]. The baseline CPIS and APACHE II scores on day 5 after the diagnosis of pneumonia (5-day CPIS and 5-day APACHE II scores) were calculated. The APACHE II score was classified as category 1, $\leq 19$; category 2,20 to 23 ; and category 3 , $\geq 24$. The CPIS score was classified as category $1, \leq 6$; category 2, 7 to 9; and category 3, $\geq 10$. Ethical approval for the study was granted by the Samsung Medical Center. The need for informed consent was waived because the study required no intervention and no breach of privacy or anonymity.

\section{Definitions}

ICU-acquired pneumonia was diagnosed on the basis of new pulmonary infiltrates plus at least two of the following criteria: fever $\geq 38^{\circ} \mathrm{C}$, blood leukocytes $\geq 10,000$ / $\mathrm{mm}^{3}$ or $\leq 3,000 / \mathrm{mm}^{3}$ and purulent tracheal secretions occurring more than 48 hours after admission to the ICU and within 72 hours of discharge from the ICU [22]. Only patients with first episodes of ICU-acquired pneumonia were eligible for the study. Appropriate antibiotic coverage was considered when at least one effective drug was included in the antibiotic treatment. Pneumonia-related deaths were considered related to the pulmonary infection if they occurred before any objective response to the antimicrobial therapy or if the pulmonary infection was considered a contributing factor to death in patients with comorbidity [3]. Each death summary was independently reviewed by two study investigators who were blinded to the use of de-escalation treatment. Mortality was classified as pneumonia-related if pneumonia was an immediate or underlying cause of death or if it played a major role in the patient's death. Mortality was defined as pneumonia-unrelated if the pneumonia was neither an immediate nor an underlying cause of death and played only a minor role, no role or an unknown role in the cause of death [23]. The overall mortality included all deaths that occurred during hospitalization.

\section{De-escalation therapy}

De-escalation was defined as streamlined antibiotic treatment driven by microbiological documentation, clinical data and the severity-of-illness index achieved by decreasing the number and/or spectrum of antibiotics. This approach to the management of ICU-acquired pneumonia involves changing the focus from the use of 
multiple agents to the use of a single agent if Pseudomonas aeruginosa is not present, shortening the therapy to $<5$ days if the culture is negative and there have been $>48$ hours of defervescence, and changing from a broad to a narrow agent in the light of culture data [24]. Accordingly, patients receiving carbapenem were deescalated to piperacillin and tazobactam, and patients receiving piperacillin and tazobactam were de-escalated to cefepime or a third-generation cephalosporin. Patients receiving combination therapy were de-escalated and switched to monotherapy by withholding fluoroquinolone, aminoglycosides or glycopeptides [14].

\section{Microbiological data collection}

Microbiological data for the patients was obtained from cultures of transendotracheal aspirates (TAs), blood, pleural fluids and bronchoalveolar lavage (BAL) fluids. A bacteriological diagnosis required one or more of the following criteria: TA cultures with $\geq 10^{5}$ colony-forming units $(\mathrm{CFU}) / \mathrm{ml}$, BAL cultures with $\geq 10^{4} \mathrm{CFU} / \mathrm{ml}$, blood or pleural fluid cultures with the same pathogen as the respiratory samples, histopathological evidence of pneumonia, positive urinary antigens of Streptococcus pneumonia or Legionella pneumophila and, for eligible specimens of TA, a white blood cell count WBC $>25$ and $<10$ epithelial cells per low-power field.

\section{Outcome criteria}

The primary outcome measure was pneumonia-related mortality at day 14 after the diagnosis of pneumonia. The secondary outcomes included overall mortality and length of mechanical ventilator support. At the time of the diagnosis of pneumonia and the initiation of deescalation therapy, the severity of illness was calculated using the APACHE II score and CPIS.

\section{Statistical analyses}

Continuous variables were compared using Student's $t$-test for normally distributed variables and the Wilcoxon rank-sum test for non-normally distributed variables. The $\chi^{2}$ statistic or Fisher's exact test was used to compare categorical variables. Multivariate analysis was performed using Cox regression analysis. The results of the statistical analysis are reported as adjusted hazard ratios (HRs) with $95 \%$ confidence intervals (95\% CIs). All $P$ values $\leq 0.05$ were considered to indicate statistical significance. PASW for Windows software package version 17.0 (SPSS, Chicago, IL, USA) was used for the analyses.

\section{Results}

One hundred thirty-seven patients (mean age, $61.0 \pm$ 16.1 years) were included in the study. Ninety-seven (70.8\%) of the patients were male. The patients had a median APACHE II score and modified CPIS of $15.0 \pm$
5.4 and $8.0 \pm 1.5$, respectively, at the time of the diagnosis of pneumonia. The median duration of mechanical ventilation and ICU stay before the diagnosis of pneumonia was $6.0 \pm 8.9$ days and $9.0 \pm 20.9$ days, respectively. The most frequent ICU admission diagnoses included general postoperative care (35.8\%), neurological diseases $(24.8 \%)$, cardiac diseases (13.1\%) and non-ICUacquired pneumonia (13.1\%).

De-escalation therapy was administered in 44 patients (32.1\%). Basic demographic and clinical characteristics of the de-escalation and non-de-escalation groups are summarized in Table 1. There were no differences in terms of prior length of ICU stay, prior antibiotic use, use of mechanical ventilation, onset of pneumonia, CCS, APACHE II score, modified CPIS and demographic characteristics between the two groups at the time of diagnosis of ICU-acquired pneumonia.

Appropriate initial antibiotics were received by 32 patients $(72.7 \%)$ in the de-escalation group and 63 patients $(67.7 \%)$ in the non-de-escalation group $(P=$ $0.55)$. Twelve patients in the de-escalation group received initial inappropriate therapy. Three patients did not receive anti-methicillin-resistant Staphylococcus aureus (anti-MRSA) antimicrobial therapy after the identification of MRSA. The therapy of three other patients was altered to include trimethoprim and sulfomethoxazole (TMP/SMX) because they had Sternotrophomonas maltophilia. Two patients showed resistance to the initial antibiotics, and four patients presented with carbapenem-resistant Gram-negative bacilli.

The rate of nonpneumonia infection during treatment of ICU-acquired pneumonia was 31.8\% (14 of 44 patients) in the de-escalation group and 23.7\% (22 of 93 patients) in the non-de-escalation group $(P=0.31)$. The median timing of de-escalation was 5.5 days. The mean modified 5-day CPIS and 5-day APACHE II score for all patients are summarized in Table 2 . The mean changes in CPIS (from the day of diagnosis of pneumonia to day 5 after the diagnosis of pneumonia) in the de-escalation and non-de-escalation groups were $1.8 \pm 1.8$ and $1.2 \pm$ 1.6 , respectively $(P=0.7)$.

There were 26 patients (19\%) with early ICU-acquired pneumonia ( $\leq 4$ days following ICU admission) and 111 patients $(81 \%)$ with late ICU-acquired pneumonia ( $\geq 5$ days following ICU admission). De-escalation was performed in $9(34.6 \%)$ of 26 patients with early ICUacquired pneumonia and in $35(31.5 \%)$ of 111 patients with late ICU-acquired pneumonia $(P=0.76)$. The rate of de-escalation was $36.5 \%$ (27 of 74 ) of the episodes in the medical ICU and $27.0 \%$ (17 of 63) in the surgical ICU $(P=0.273)$.

Of the 137 patients, 117 microbiological pathogens were isolated from 109 patients (79.6\%). The initial microbiological identification was based on quantitative 
Table 1 Clinical characteristics of the de-escalation group and the non-de-escalation group in patients with ICUacquired pneumonia ${ }^{a}$

\begin{tabular}{|c|c|c|c|}
\hline Characteristics & De-escalation group $(N=44)$ & Non-de-escalation group $(N=93)$ & $P$ value \\
\hline Mean age $( \pm S D)$, yr & $57.45 \pm 17.5$ & $59.02 \pm 15.4$ & 0.45 \\
\hline Male:female ratio, $n$ & 30:14 & $67: 26$ & 0.64 \\
\hline \multicolumn{4}{|l|}{ Underlying conditions, $n$ (\%) } \\
\hline Diabetes mellitus & $8(18.2 \%)$ & $14(15.0 \%)$ & \\
\hline Structural lung disease & $4(9.1 \%)$ & $9(9.7 \%)$ & \\
\hline Renal failure $(\mathrm{Cr}>2.0 \mathrm{mg} / \mathrm{dl})$ & $4(9.1 \%)$ & $19(20.4 \%)$ & \\
\hline Malignancy & $10(22.7 \%)$ & $34(36.6 \%)$ & \\
\hline Liver disease & $4(9.1 \%)$ & $8(8.6 \%)$ & \\
\hline Transplantation & $5(11.4 \%)$ & $8(8.6 \%)$ & \\
\hline Congestive heart failure & $10(22.7 \%)$ & $15(16.1 \%)$ & \\
\hline Postoperative state & $24(54.5 \%)$ & $59(63.4 \%)$ & \\
\hline Cerebrovascular accident & $12(27.3 \%)$ & $22(23.7 \%)$ & \\
\hline Alcoholism & $1(2.3 \%)$ & $3(3.2 \%)$ & \\
\hline Prior antibiotic use, $n(\%)$ & $42(95.9 \%)$ & $86(92.5 \%)$ & 0.51 \\
\hline Mean prior length of ICU stay $( \pm S D)$, days & $13.43 \pm 22.7$ & $13.39 \pm 20.0$ & 0.88 \\
\hline Use of $M V, n(\%)$ & $39(88.6 \%)$ & $85(91.4 \%)$ & 0.60 \\
\hline Mean prior length of $\mathrm{MV}( \pm \mathrm{SD})$, days & $7.6 \pm 6.4$ & $7.9 \pm 9.8$ & 0.37 \\
\hline Mean CCS $( \pm S D)$ & $2.43 \pm 1.5$ & $2.34 \pm 1.5$ & 0.66 \\
\hline Mean APACHE II score $( \pm S D)$ & $15.6 \pm 5.5$ & $15.3 \pm 5.3$ & 0.90 \\
\hline Mean CPIS $( \pm$ SD) & $8.32 \pm 1.6$ & $8.46 \pm 1.3$ & 0.06 \\
\hline
\end{tabular}

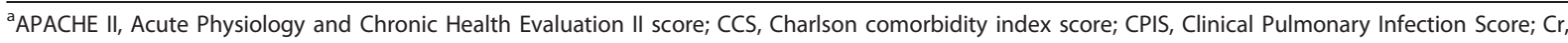
creatinine; ICU, intensive care unit; MV, mechanical ventilation; SD, standard deviation.

information from the TA in 99 cases (73.8\%), BAL fluid samples in 5 cases (3.6\%), and blood cultures in 3 cases (2.2\%). MRSA was the most common pathogen identified $(n=44,37.6 \%)$, followed by Acinetobacter spp. (16.2\%), Pseudomonas spp. (15.4\%), Klebsiella pneumoniae $(10.3 \%)$ and S. maltophilia (6.8\%).

De-escalation was performed in $32(29.4 \%)$ of 109 patients with positive cultures and in $12(42.9 \%)$ of 28 patients with negative cultures. De-escalation was initiated in $34.5 \%$ of episodes with potentially resistant pathogens (nonfermenting Gram-negative bacilli (NFGNB) and MRSA) compared to $65.5 \%$ among the remaining pathogens $(P=0.45)$. Among the 95 patients in whom the antibiotics could be de-escalated, based on the antimicrobial susceptibility data, 32 patients (33.7\%) received de-escalation therapy.

The most frequently prescribed empirical antibiotic was carbapenem $(35 \%)$, followed by piperacillin and tazobactam (28.5\%), third-generation cephalosporin $(22.6 \%)$ and cefepime (6.6\%). Vancomycin was prescribed in 60 cases $(43.8 \%)$ as part of a combination regimen. The proportion of cases with appropriate initial antibiotic treatment was $69.3 \%$ (95 of 137). A two-agent combination regimen was prescribed in $103(75.2 \%)$ of

Table 2 Comparison of the APACHE II score and the modified CPIS at day 5 of pneumonia diagnosis between the two groups $^{\mathbf{a}}$

\begin{tabular}{lccc}
\hline Severity index & De-escalation & Non-de-escalation & $P$ value \\
\hline Mean APACHE II score $( \pm$ SD) & $13.6 \pm 4.4$ & $15.8 \pm 6.0$ & 0.03 \\
APACHE II score, $n(\%)$ & & & 0.04 \\
$\quad<19$ & $34(87.2 \%)$ & $55(72.4 \%)$ & $11(14.5 \%)$ \\
$\quad 19$ to 23 & $4(10.3 \%)$ & $10(13.2 \%)$ & $7.5 \pm 1.4$ \\
$\quad>23$ & $1(2.6 \%)$ & & 0.002 \\
Mean CPIS $( \pm$ SD) & $6.5 \pm 1.2$ & $25(27.8 \%)$ & 0.009 \\
CPIS category, $n(\%)$ & & $61(67.8 \%)$ & \\
$\quad 4$ to 6 & $21(48.8 \%)$ & $4(2.7 \%)$ & \\
7 to 9 & $22(51.2 \%)$ & $0(0 \%)$ & \\
\hline 10 &
\end{tabular}

${ }^{a}$ APACHE II, Acute Physiology and Chronic Health Evaluation II; CPIS, Clinical Pulmonary Infection Score. 
the total group of patients: 37 in the de-escalation group and 66 in the non-de-escalation group. Monotherapy was prescribed in $32(23.4 \%)$ of all patients: 7 in the deescalation group and 25 in the non-de-escalation group. Two patients received three antibiotics as empirical therapy. There was no difference between the groups with regard to the use of combination therapy versus monotherapy.

De-escalation therapy in 44 patients was implemented by decreasing the number of antibiotics, the spectrum of antibiotics or both. The number of antibiotics used was decreased for 25 patients (56.8\%). In the majority of cases, vancomycin was discontinued when MRSA was not identified. Antibiotics were streamlined to a narrow spectrum in 12 patients $(27.3 \%)$, whereas 7 patients (15.9\%) received de-escalation therapy by decreasing the number as well as the spectrum of antibiotics. Thirtyfour of the 137 patients died during a 30-day follow-up period. This represented an overall mortality rate of $24.8 \%$ (34 of 137) and a pneumonia-related mortality rate of $10.2 \%$ (14 of 137).

Although there was a lower trend for the pneumoniarelated mortality rate in the de-escalation group, the difference did not reach statistical significance by day 14 after the diagnosis of ICU-acquired pneumonia $(1(2.3 \%)$ of 44 patients versus $10(10.8 \%)$ of 93 patients; $P=0.08)$ (Figure 1). The pneumonia-related mortality at day 30 was significantly lower in the de-escalation group than in the non-de-escalation group (1 $(2.3 \%)$ of 44 patients versus 13 (14\%) of 93 patients; $P=0.03$ ) (Figure 1). With regard to overall mortality, the de-escalation group had a significantly lower mortality rate than the non-de-escalation group by day $14(P=0.04)$ and by day $30(P=0.01)$.

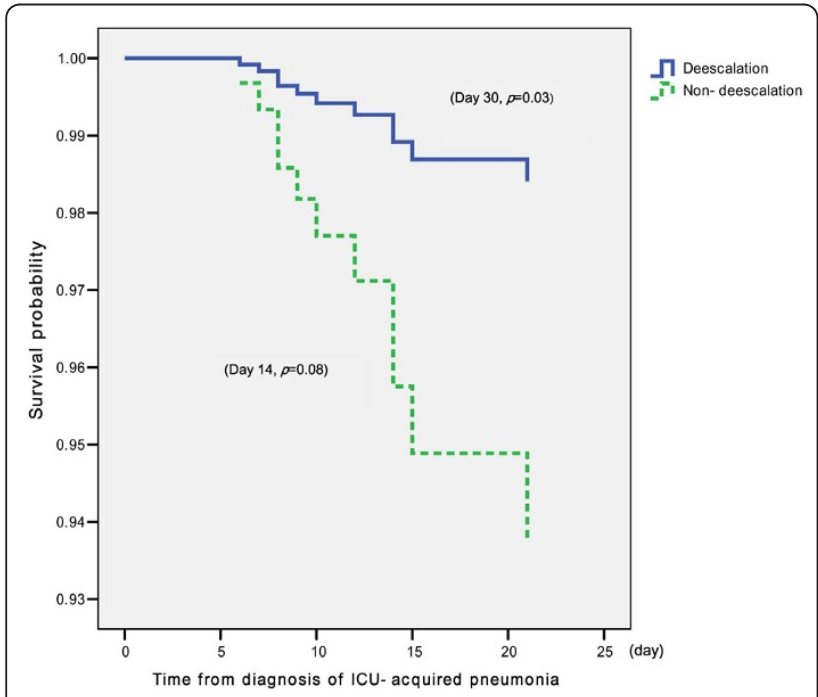

Figure 1 Pneumonia-related mortality between the deescalation group and the non-de-escalation groups.
The pneumonia-related mortality was associated with inadequate empirical antibiotics, non-de-escalation of antibiotics, baseline APACHE II score, 5-day APACHE II score and 5-day CPIS on the basis of univariate analysis. However, only the 5-day APACH E II score and the 5-day CPIS were found to be independent risk factors associated with pneumonia-related mortality and overall mortality (Table 3).

The APACHE II score and the modified CPIS in patients with negative cultures are outlined in Table 4. There was a downward trend in the scores of the patients in the de-escalation group; however, the difference was not statistically significant when compared to the non-de-escalation group. The 30 -day pneumoniarelated mortality of patients with negative cultures was 0 of $12(0 \%)$ in the de-escalation group and 2 of 16 $(12.5 \%)$ in the non-de-escalation group. Two patients with negative cultures died on day 3 and day 25, respectively, and they had relatively high APACHE II scores and modified CPIS on day 5.

\section{Discussion}

In this study, the overall de-escalation rate was $32.1 \%$ (44 of 137 patients). This proportion increased to $33.7 \%$ (32 of 95 patients) when only episodes in which de-escalation was applicable based on antimicrobial susceptibility were considered. The de-escalation rate in this study was lower than the rates in previous studies, in which the deescalation rates of patients with susceptible pathogens have been reported to be $38 \%$ to $51.9 \%$ [11,14]. AlvarenzLerma et al. [11] reported that a low de-escalation rate is probably due to a high prevalence of Pseudomonas spp. infection (30.6\%), intensive prior use of antibiotics (79.1\%) and a larger proportion of late-onset episodes $(90.6 \%)$. Our study has shown a high incidence of NFGNB cases, including Pseudomonas spp. infection (38.4\%) and MRSA infection (37.6\%). In addition, a high rate of prior use of antibiotics (93.4\%) and a larger proportion of cases with late ICU-acquired pneumonia (81\%) were evident in our study. The low de-escalation rate in this study might be due to these factors.

Currently, de-escalation is significantly less frequent in patients with pneumonia who have NFGNB and MRSA. This is because the effectiveness of this approach varies according to local patterns of antibiotic sensitivity. Although Korea has a relatively high prevalence of multiresistant pathogens $[25,26]$, the de-escalation rate in patients with NFGNB and MRSA in the present study was $34.5 \%$, which is much higher than the $2.7 \%$ and $23.1 \%$ rates described in previous reports $[11,14]$.

Among 44 patients in the de-escalation group, 12 patients received inappropriate initial antibiotics. Two patients were changed to narrow-spectrum antibiotics because they showed resistance to initial antibiotics. 
Table 3 Factors associated with 30-day pneumonia-related mortality in patients with ICU-acquired pneumonia determined by multivariable analysis ${ }^{\mathrm{a}}$

\begin{tabular}{|c|c|c|c|}
\hline Variable & Adjusted hazard ratio ${ }^{b}$ & $95 \% \mathrm{Cl}$ & $P$ value \\
\hline Inadequacy of antibiotics & 2.145 & 0.483 to 9.536 & 0.316 \\
\hline Non-de-escalation of antibiotics & 3.988 & 0.047 to 6.985 & 0.245 \\
\hline Baseline APACHE II score (reference score <19) & & & 0.198 \\
\hline 20 to 23 & 2.528 & 0.609 to 10.493 & 0.201 \\
\hline$\geq 24$ & 7.611 & 0.615 to 94.179 & 0.114 \\
\hline 5-day APACHE II score (reference score <19) & & & 0.011 \\
\hline 20 to 23 & 4.934 & 0.974 to 25.003 & 0.054 \\
\hline$\geq 24$ & 12.839 & 2.359 to 69.883 & 0.003 \\
\hline 5-day CPIS (reference score 4 to 6) & & & 0.017 \\
\hline 7 to 9 & 2.154 & 0.361 to 12.861 & 0.400 \\
\hline$\geq 10$ & 26.782 & 2.180 to 329.011 & 0.010 \\
\hline
\end{tabular}

${ }^{\mathrm{a}} \mathrm{ICU}$, intensive care unit; $95 \% \mathrm{Cl}, 95 \%$ confidence interval; APACHE II, Acute Physiology and Chronic Health Evaluation II; CPIS, Clinical Pulmonary Infection Score; ${ }^{\mathrm{b}} \mathrm{Cox}$ proportional hazard regression analysis was used to determine the relationship between mortality and independent baseline variables identified in univariable analysis, including inadequacy of initial antibiotics, de-escalation of antibiotics, baseline APACHE II SCore, 5-day APACHE II score and 5-day CPIS.

Four patients who harbored carbapenem-resistant, Gram-negative bacilli received carbapenem or piperacillin and tazobactam, because colistin was not available at that time. Two of these patients died.

Three patients did not receive anti-MRSA antimicrobial agents, even though MRSA infection was identified, because the patients were improving with initial antibiotics. All three patients survived. The MRSA isolated from quantitative tracheal aspirate might not be true pathogens. If the bacteria were merely colonizing pathogens, but not the cause of infection, then the initial antimicrobial therapy would be inappropriate. Quantitative TA allows the identification of pathogens in the great majority (90\%) of cases [14]. The antibiotic regimen of three other patients with $S$. maltophilia was changed to include TMP/SMX and the broad-spectrum antibiotics were switched to narrow-spectrum antibiotics.

The pneumonia-related mortality rate was not significantly different in the de-escalation group compared to the non-de-escalation group at day $14(P=0.08)$. The pneumonia-related mortality and overall mortality at day 30, however, was significantly lower in the de-escalation group $(P=0.03)$. This finding is consistent with the results reported by Kollef et al. [13]. The pneumonia-related mortality was associated with the 5-day APACHE II score and the 5-day CPIS, even with the adjustment for other factors in addition to the scores at baseline in this study.

The 5-day APACHE II score and the 5-day CPIS were significantly lower in the de-escalation group compared to the non-de-escalation group. The cause of high mortality in the non-de-escalation group was probably related to the high APACHE II score and modified CPIS on day 5, as well as the timing of de-escalation (Table 2). This suggests that de-escalation is effective in patients with ICU-acquired pneumonia who have a more stable severity-of-illness index on days 3 to 5 after the diagnosis of pneumonia. The mean changes in CPIS in the de-escalation group were higher, although this result was statistically insignificant, than in the non-de-escalation group. De-escalation was not performed in patients with a category 3 APACHE II score and CPIS at the time of deescalation. However, patients in category 1 can be safely de-escalated, and those in category 2 can be considered for de-escalation on the basis of the clinical response of the pneumonia.

Table 4 Comparison of severity-of-illness index in patients with negative cultures ${ }^{\text {a }}$

\begin{tabular}{|c|c|c|c|}
\hline Severity index & De-escalation group $(N=12)$ & Non-de-escalation group $(N=16)$ & $P$ value \\
\hline Mean baseline APACHE II score $( \pm S D)$ & $15.36 \pm 5.9$ & $16.56 \pm 6.6$ & 0.63 \\
\hline Mean baseline CPIS ( \pm SD) & $6.83 \pm 1.2$ & $6.56 \pm 0.9$ & 0.50 \\
\hline 5-day APACHE II score $( \pm S D)$ & $11.70 \pm 5.0$ & $13.82 \pm 3.3$ & 0.26 \\
\hline Category 1, n (\%) & 11 of $12(91.7 \%)$ & 14 of $16(87.5 \%)$ & 0.90 \\
\hline Category 2, n (\%) & 1 of $12(8.3 \%)$ & 2 of $16(12.5 \%)$ & \\
\hline Mean 5-day CPIS ( \pm SD) & $5.9 \pm 1.1$ & $6.5 \pm 1.2$ & 0.23 \\
\hline Category 1, n (\%) & 9 of $12(75 \%)$ & 8 of $16(50 \%)$ & 0.13 \\
\hline Category 2, n (\%) & 3 of $12(25 \%)$ & 8 of $16(50 \%)$ & \\
\hline
\end{tabular}

${ }^{a}$ APACHE II, Acute Physiology and Chronic Health Evaluation II; CPIS, Clinical Pulmonary Infection Score. 
De-escalation was carried out in $12(42.9 \%)$ of 28 patients with negative cultures, which resulted in no mortality. Rello et al. [14] did not perform de-escalation in patients with negative cultures because of concern about false-negative cultures and the time to resolution of the febrile illness. Alvarenz-Lerma et al. [11] also did not perform de-escalation in 113 patients with negative cultures, which resulted in a prolonged administration of imipenem. The high portion of patients with negative cultures who did not receive de-escalation was probably influenced by the lack of specific recommendations for de-escalation.

Although our data were collected from a small number of patients with negative cultures, more than $40 \%$ of the patients received de-escalation therapy, and all 12 patients survived at day 30 after the diagnosis of pneumonia. The de-escalation group had slightly lower APACHE II score and a lower modified CPIS trend at the time of de-escalation compared to the non-deescalation group, although both were not statistically significant. Among all patients with negative cultures, two patients in the non-de-escalation group died. They had category 3 APACHE II scores and modified CPIS. If patients with negative cultures are classified in category 1 of the APACHE II score and modified CPIS by day 5 , the findings of this study suggest that de-escalation could be considered for such patients with ICUacquired pneumonia. This is the first study to show that de-escalation therapy in patients with negative cultures is feasible in patients with stable APACHE II scores and modified CPIS.

This study has several limitations. First, because it was a retrospective cohort study, there was no control group. It is ethically impossible to randomize ICU patients because patients with ICU-acquired pneumonia are seriously ill and require complex care. These limitations were minimized by the multivariate analysis. Second, it is difficult to distinguish true infection from colonization. Our respiratory tract specimens were mainly TA (73.8\%). It has been noted above that three MRSA isolates in the de-escalation group might not have been true pathogens. It is somewhat confusing to say that therapy was inappropriate because it is possible that MRSA was a colonizer. However, we performed microbiological identification using quantitative methods, and the number of BAL specimens from patients was similar to those described in previous reports. Rello et al. [14] reported that quantitative TA and bronchoscopic samples allowed identification of pathogens in $90 \%$ and $93 \%$ of episodes, respectively, and there were no differences between the patients diagnosed on the basis of quantitative TA or bronchoscopy with regard to crude mortality and ICU mortality data. Third, there are no standard criteria for initial empirical antibiotic treatments and the timing of de-escalation. However, clinicians usually attempt to follow the currently available ATS and IDSA VAP guidelines for the diagnosis and treatment of pneumonia. Fourth, because of the small number of patients and because it was a singlecenter study, the results might not be applicable to other groups. Fifth, we measured the modified clinical pulmonary infection score retrospectively. The scores of tracheal secretions were classified as 0 (rare), 1 (abundant) and 2 (abundant and purulent) using ICU nursing records [20]. The ICU nurses recorded the hourly full particles of patients' information. For example, tracheal secretions were recorded as scant whitish, large whitish or large yellowish secretions. We reviewed the nursing records and decided on a score of tracheal secretions.

\section{Conclusions}

The patients in the de-escalation group did not show increased mortality compared to those in the non-deescalation group. The results of this study suggest that de-escalation therapy based on the APACHE II score and the modified CPIS 5 days after the diagnosis of pneumonia can be safely applied with good clinical outcomes for patients with ICU-acquired pneumonia, even in those with negative cultures. Prospective, large studies are needed to evaluate the efficacy of the de-escalation strategy in patients with negative cultures.

\section{Key messages}

- The initial choice of antimicrobial therapy is critical to the clinical outcomes of patients with nosocomial pneumonia.

- Awareness of the need for early and appropriate therapy may tempt the clinician to use aggressive empirical therapy at the first sign of infection.

- De-escalation therapy is a method currently used for the management of serious infections, especially in patients with nosocomial pneumonia.

- De-escalation therapy based on APACHE II score and the modified CPIS 5 days after the diagnosis of pneumonia can be safely applied with good clinical outcomes among patients with ICU-acquired pneumonia, even in those patients with negative cultures. - Prospective, large studies are needed to evaluate the efficacy of the de-escalation strategy in patients with ICU-acquired pneumonia.

\section{Abbreviations}

APACHE II: Acute Physiology and Chronic Health Evaluation II; ATS: American Thoracic Society; CCS: Charlson comorbidity index score; Cl: confidence interval; CPIS: Clinical Pulmonary Infection Score; HR: hazard ratio; IDSA: Infectious Diseases Society of America; MRSA: methicillin-resistant Staphylococcus aureus; MV: mechanical ventilation; NFGNB: nonfermenting Gram-negative bacilli; VAP: ventilator-associated pneumonia. 


\section{Acknowledgements}

We thank all of the nurses and doctors in the participating ICUs for their support in this study.

This study was supported by grant A102065 from the Korea Health 21 R\&D Project, Ministry of Health, Welfare \& Family Affairs, Republic of Korea.

\section{Author details}

${ }^{1}$ Division of Infectious Diseases, Samsung Medical Center, Sungkyunkwan University School of Medicine, 50 Ilwon-dong, Gangnam-gu, Seoul 135-710, Republic of Korea. ${ }^{2}$ Division of Infectious Diseases, Konyang University Hospital, 685 Gasuwon-dong, Seo-gu, Daejeon 302-718, Republic of Korea. ${ }^{3}$ Division of Infectious Diseases, Kyunghee University Hospital, Hoegi-dong, Dongdaemun-gu, Seoul 130-702, Republic of Korea. ${ }^{4}$ Division of Infectious Diseases, Konkuk University Hospital, 4-12 Hwawang-dong, Gangjin-gu, Seoul 143-701, Republic of Korea. ${ }^{5}$ Division of Infectious Diseases, Chungnam National University Hospital, 33 Munhwa-ro, Jung-gu, Daejeon 301-721, Republic of Korea. ${ }^{6}$ Divisions of Pulmonary and Critical Care Medicine, Samsung Medical Center, Sungkyunkwan University School of Medicine, 50 Ilwon-dong, Gangnam-gu, Seoul 135-710, Republic of Korea. ${ }^{7}$ Asian-Pacific Research Foundation for Infectious Diseases, 50 Ilwon-dong, Gangnam-gu, Seoul 135-710, Republic of Korea.

\section{Authors' contributions}

MKJ, GYS and KRP contributed to study conception and design. MKJ, JL, HSC, SYM, EJJ, YEH, KMS and SMC collected and analyzed the data. MKJ drafted the manuscript. MKJ, SMC, JHS and KRP participated in the drafting and revision of the manuscript. All authors were involved in data acquisition and read and approved the final manuscript.

\section{Competing interests}

The authors declare that they have no competing interests.

Received: 23 April 2010 Revised: 9 February 2011

Accepted: 2 March 2011 Published: 2 March 2011

\section{References}

1. Alvarez-Lerma F: Modification of empiric antibiotic treatment in patients with pneumonia acquired in the intensive care unit. ICU-Acquired Pneumonia Study Group. Intensive Care Med 1996, 22:387-394.

2. Luna CM, Vujacich P, Niederman MS, Vay C, Gherardi C, Matera J, Jolly EC: Impact of BAL data on the therapy and outcome of ventilatorassociated pneumonia. Chest 1997, 111:676-685

3. Rello J, Gallego M, Mariscal D, Soñora R, Valles J: The value of routine microbial investigation in ventilator-associated pneumonia. Am J Respir Crit Care Med 1997, 156:196-200.

4. Celis R, Torres A, Gatell JM, Almela M, Rodríguez-Roisin R, Agustí-Vidal A: Nosocomial pneumonia: a multivariate analysis of risk and prognosis. Chest 1988, 93:318-324.

5. Ibrahim EH, Sherman G, Ward S, Fraser VJ, Kollef MH: The influence of inadequate antimicrobial treatment of bloodstream infections on patient outcomes in the ICU setting. Chest 2000, 118:146-155.

6. Iregui M, Ward S, Sherman G, Fraser VJ, Kollef MH: Clinical importance of delays in the initiation of appropriate antibiotic treatment for ventilatorassociated pneumonia. Chest 2002, 122:262-268.

7. Kollef MH, Sherman G, Ward S, Fraser VJ: Inadequate antimicrobial treatment of infections: a risk factor for hospital mortality among critically ill patients. Chest 1999, 115:462-474.

8. Leroy O, Meybeck A, d'Escrivan T, Devos P, Kipnis E, Georges H: Impact of adequacy of initial antimicrobial therapy on the prognosis of patients with ventilator-associated pneumonia. Intensive Care Med 2003, 29:2170-2173.

9. Torres A, Aznar R, Gatell JM, Jiménez P, González J, Ferrer A, Celis R, Rodriguez-Roisin R: Incidence, risk, and prognosis factors of nosocomial pneumonia in mechanically ventilated patients. Am Rev Respir Dis 1990, 142:523-528.

10. Hoffken G, Niederman MS: Nosocomial pneumonia: the importance of a de-escalating strategy for antibiotic treatment of pneumonia in the ICU. Chest 2002, 122:2183-2196.

11. Alvarez-Lerma F, Alvarez B, Luque P, Ruiz F, Dominguez-Roldan JM, Quintana E, Sanz-Rodriguez C, ADANN Study Group: Empiric broad- spectrum antibiotic therapy of nosocomial pneumonia in the intensive care unit: a prospective observational study. Crit Care 2006, 10:R78.

12. Kollef $M H$, Kollef KE: Antibiotic utilization and outcomes for patients with clinically suspected ventilator-associated pneumonia and negative quantitative BAL culture results. Chest 2005, 128:2706-2713.

13. Kollef MH, Morrow LE, Niederman MS, Leeper KV, Anzueto A, Benz-Scott L, Rodino FJ: Clinical characteristics and treatment patterns among patients with ventilator-associated pneumonia. Chest 2006, 129:1210-1218.

14. Rello J, Vidaur L, Sandiumenge A, Rodríguez A, Gualis B, Boque C, Diaz E: De-escalation therapy in ventilator-associated pneumonia. Crit Care Med 2004, 32:2183-2190.

15. Singh N, Rogers P, Atwood CW, Wagener MM, Yu VL: Short-course empiric antibiotic therapy for patients with pulmonary infiltrates in the intensive care unit: a proposed solution for indiscriminate antibiotic prescription. Am J Respir Crit Care Med 2000, 162:505-511.

16. Micek ST, Ward S, Fraser VJ, Kollef MH: A randomized controlled trial of an antibiotic discontinuation policy for clinically suspected ventilatorassociated pneumonia. Chest 2004, 125:1791-1799.

17. Soo Hoo GW, Wen YE, Nguyen TV, Goetz MB: Impact of clinical guidelines in the management of severe hospital-acquired pneumonia. Chest 2005, 128:2778-2787.

18. American Thoracic Society; Infectious Diseases Society of America: Guidelines for the management of adults with hospital-acquired, ventilator-associated, and healthcare-associated pneumonia. Am J Respir Crit Care Med 2005, 171:388-416.

19. Knaus WA, Draper EA, Wagner DP, Zimmerman JE: APACHE II: a severity of disease classification system. Crit Care Med 1985, 13:818-829.

20. Fartoukh M, Maitre B, Honoré S, Cerf C, Zahar JR, Brun-Buisson C: Diagnosing pneumonia during mechanical ventilation: the clinical pulmonary infection score revisited. Am J Respir Crit Care Med 2003, 168:173-179.

21. Charlson ME, Pompei $P$, Ales KL, Mackenzie CR: A new method of classifying prognostic comorbidity in longitudinal studies: development and validation. J Chronic Dis 1987, 40:373-383.

22. Garner JS, Jarvis WR, Emori TG, Horan TC, Hughes JM: CDC definitions for nosocomial infections, 1988. Am J Infect Control 1988, 16:128-140.

23. World Health Organization: Manual of the International Statistical Classification of Diseases, Injuries, and Causes of Death Geneva, Switzerland: World Health Organization; 1977.

24. Vidaur L, Sirgo G, Rodríguez AH, Rello J: Clinical approach to the patient with suspected ventilator-associated pneumonia. Respir Care 2005, 50:965-974.

25. Lee K, Lim CH, Cho JH, Lee WG, Uh Y, Kim HJ, Yong D, Chong Y: High prevalence of ceftazidime-resistant Klebsiella pneumoniae and increase of imipenem-resistant Pseudomonas aeruginosa and Acinetobacter spp. in Korea: a KONSAR program in 2004. Yonsei Med J 2006, 47:634-645.

26. Chong $Y$, Lee K: Present situation of antimicrobial resistance in Korea. $J$ Infect Chemother 2000, 6:189-195.

doi:10.1186/cc10072

Cite this article as: Joung et al:: Impact of de-escalation therapy on clinical outcomes for intensive care unit-acquired pneumonia. Critical Care 2011 15:R79.

\section{Submit your next manuscript to BioMed Central and take full advantage of:}

- Convenient online submission

- Thorough peer review

- No space constraints or color figure charges

- Immediate publication on acceptance

- Inclusion in PubMed, CAS, Scopus and Google Scholar

- Research which is freely available for redistribution

Submit your manuscript at www.biomedcentral.com/submit
C Biomed Central 